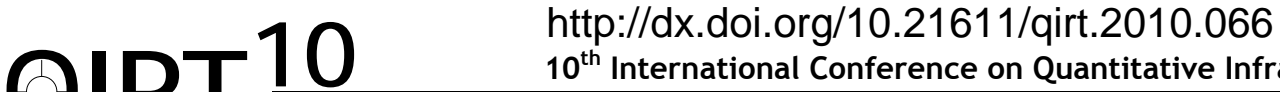 \\ $10^{\text {th }}$ International Conference on Quantitative InfraRed Thermography \\ July 27-30, 2010, Québec (Canada)
}

\section{A hybrid pulsed thermography processing technique for the depth estimation of subsurface defects combining TSR and PPT}

\author{
by M. Klein, A. Bendada, C. Ibarra-Castanedo and X. P. V. Maldague
}

Computer Vision and Systems Laboratory, Department of Electrical and Computer Engineering, 1065, av. de la Médecine, Laval University, Quebec City, Canada, G1V 0A6; \{Bendada, IbarraC, MaldagX\}@gel.ulaval.ca

\begin{abstract}
A hybrid approach, combining thermographic signal reconstruction (TSR) and pulsed phase thermography (PPT), is proposed and tested for the depth estimation of subsurface defects from pulsed thermographic data. First, TSR is used to obtain the polynomial coefficients from raw data. A synthetic thermogram sequence is then reconstructed from the obtained coefficients. Finally, quantitative PPT is applied to this sequence for defect depth estimation purposes. This combined procedure allows to considerably reduce signal noise, improving depth estimation results as a consequence. A TSR module has been added to an existent graphical user interface (ir_view) to perform these operations.
\end{abstract}

\section{Introduction}

Pulsed thermography is without a doubt an interesting nondestructive testing and evaluation (NDT\&E) technique that is gaining popularity thanks to its easiness of deployment and to the development of suitable commercial equipment. Several processing techniques are available to improve defect contrast and signal-to-noise ratio. TSR is one of such techniques that also posses other interesting advantages such as data compression and noise reduction, and that confers as well the possibility to produce analytical results, e.g. first and second time derivatives [1, 2]. PPT is another attractive technique that transforms the time data to the frequency domain in order to recover the amplitude and phase information, the later normally being less affected by optical and thermographic artifacts than raw thermal data [3]. A quantitative PPT algorithm has been proposed to estimate the depth of subsurface defects from the phase [4]. However, noise content in the phase is considerable. Hence, a de-noising step is often required in order to produce suitable results. In this paper, a hybrid approach combining the de-noising capabilities of TSR to the depth retrieval PPT algorithm, is proposed to improve the depth estimation results. An existing graphical user interface named ir_view is already available to perform PPT calculations, among other processing techniques [5]. Two processing modules have been added to this interface for this investigation: (1) a TSR module allowing to calculate the polynomial coefficients and to synthetically reconstruct the signal, and (2) a quantitative PPT module for depth estimation.

\section{Thermographic signal reconstruction (TSR)}

Thermographic signal reconstruction (TSR) [1] is an attractive technique that allows increasing spatial and temporal resolution of a thermogram sequence, while reducing at the same time the amount of data to be manipulated. TSR is based on the assumption that temperature profiles for non-defective pixels should follow the decay curve given by the onedimensional solution of the Fourier equation, which in the double-logarithmic form follows a linear decay with time. To fit the thermographic data, Shepard 2001 proposed to use a $p$-degree polynomial of the form:

$$
\ln (\Delta T)=a_{0}+a_{1} \ln (t)+a_{2} \ln ^{2}(t)+\ldots+a_{p} \ln ^{p}(t)
$$

Thermal profiles corresponding to non-defective areas in the sample will follow an approximately linear decay, while the thermal behaviour of a defective area will diverge from linearity. Typically, $p$ is set to 4 or 5 to avoid "ringing" and to insure a good correspondence between acquired data and fitted values. At the end, the entire raw thermogram sequence is reduced to $p+1$ coefficient images (one per polynomial coefficient) from which synthetic thermograms can be reconstructed.

Synthetic data processing brings interesting advantages such as: significant noise reduction, the possibility for analytical computations and data compression (from $N$ to $p+1$ images). Analytical processing provides the possibility of estimating the actual thermogram for a time between acquisitions from the polynomial coefficients. Furthermore, calculation of first and second time derivatives from the synthetic coefficients is straightforward. First time derivatives indicate the rate of cooling while second time derivatives refer to the rate of change in the rate of cooling. Therefore, time derivatives are more sensitive to temperature changes than raw thermal images. Finally, TSR synthetic data can be used in combination with P PT to perform quantitative analysis as described later. 


\section{Pulsed phase thermography (PPT)}

In pulsed phase thermography (PPT) data is transformed from the time domain to the frequency domain using the one-dimensional discrete Fourier transform (DFT), real and imaginary parts of the complex transform are used to estimate the amplitude $A$, and the phase $\varphi$ [3]. The phase is of particular interest in NDE given that it is less affected than raw thermal data by environmental reflections, emissivity variations, non-uniform heating, and surface geometry and orientation. These phase characteristics are very attractive not only for qualitative inspections but also for quantitative characterization of materials. For instance, a depth inversion technique using the phase from PPT has been proposed [4]. The depth of a defect can be calculated from a relationship of the form [4]:

$$
z=C_{1} \cdot \mu=C_{1} \sqrt{\frac{\alpha}{\pi \cdot f_{b}}}
$$

where $f_{b}[\mathrm{~Hz}]$ is the blind frequency defined as the limiting frequency at which a defect located at a particular depth presents enough (phase or amplitude) contrast to be detected on the frequency spectra. Defect contrast is enhanced using the phase (given the phase properties described above) allowing deeper probing. Conventional experimental $C 1$ values when using the phase from lock-in thermography experiments range between 1.5 to more than 2 with a value of $\mathrm{C} 1=1.82$ (Thomas et al., 1980) typically adopted in experimental studies. P PT results agree with these numbers for homogeneous materials: C1 1.72 for steel, and C1 2.0 for Plexiglas ${ }^{\circledR}$, and for composite materials: C 1 1.73 for CFRP. In this way, the inversion problem in PPT is reduced to the estimation of fb from the phase.

It is well-known that noise content present in phase data is considerable, especially at high frequencies. This causes a problem for the determination of the blind frequency. A de-noising step is therefore often required. The combination of PPT and TSR has proven to be very effective for this matter, reducing noise and allowing the depth retrieval for a defect.

\section{Results and discussion}

Results will be presented and discussed during the Conference.

\section{REFERENCES}

[1] Shepard S. M., Lhota J. R., Rubadeux B. A., Ahmed T., W ang D. "Enhancement and reconstruction of thermographic NDT data", Proc. SPIE - The International Society for Optical Engineering, Thermosense XXIV, Orlando, FL, 2002, Eds. X. P. Maldague and A. Rozlosnik, 4710:531-535.

[2] Shepard S. M., Lhota J. R., Rubadeux B. A., Ahmed T., Wang D. "Enhancement and reconstruction of thermographic NDT data", Proc. SPIE - The International Society for Optical Engineering, Thermosense XXIV, Orlando, FL, 2002, Eds. X. P. Maldague and A. Rozlosnik, 4710:531-535.

[3] Maldague X. P. and Marinetti S. "Pulse Phase Infrared Thermography," J . Appl. Phys., 79(5):2694-2698, 1996.

[4] Ibarra-Castanedo C. "Quantitative subsurface defect evaluation by pulsed phase thermography: depth retrieval with the phase," Ph. D. thesis, Laval University, 2005. [accessible online: http://www.theses.ulaval.ca/2005/23016/23016.pdf].

[5] Klein M., Pilla M. and Maldague X. "IR_View: A graphical user interface to process infrared images with MATLAB," application and documentation available online at: http://irview.m-klein.com (accessed February 21st 2007). 